\title{
Linear to turbulent Görtler instability transition
}

\author{
J. Dagaut, ${ }^{1}$ M.E. Negretti, ${ }^{1,}$ a) G. Balarac, ${ }^{1,2}$ and C. Brun ${ }^{1}$ \\ 1)Univ. Grenoble Alpes, CNRS, Grenoble INP, LEGI, 38000, Grenoble, \\ France \\ ${ }^{2)}$ Institut Univ. de France, 1 rue Descartes, 75231 Paris, France
}

(Dated: 8 October 2021)

We present results from a high resolved Large Eddy Simulation of a freely developing Blasius profile over a concave boundary in a large spanwise domain. Due to the large initial Reynolds and Görtler numbers $\left(\operatorname{Re}_{\theta, 0}=1170, G_{\theta, 0}=75\right)$, we observe the onset of two dominant wavelengths, the first dominating in the linear/transition region, $\lambda_{1}$, and the second dominating in the turbulent region, $\lambda_{2}$. Extending previous linear stability analysis (LSA) to higher Görtler numbers and non-dimensional wavenumbers, both dominant wavelengths of the Görtler instability correspond to predictions of LSA, the latter comparable to laminar theory by replacing the kinematic viscosity with the turbulent viscosity in the definition of the Görtler number. The predicted spatial modes compare well with the computed profiles for both $\lambda_{1}$ and $\lambda_{2}$. The skin friction coefficient $C_{f}$ is found heterogeneous in the spanwise direction according to the emerging wavelengths $\lambda_{1}$ and $\lambda_{2}$ of the Görtler instability. We report a smooth increase of $C_{f}$ from the theoretical predictions of a laminar boundary layer to those for a turbulent boundary layer over a flat plate with a slight overshoot in the domain of existence of the second dominant wavelength $\lambda_{2}$.

a)Electronic mail: eletta.negretti@legi.cnrs.fr 


\section{INTRODUCTION}

Boundary layer flows are ubiquitous in both nature and engineering applications and are subject to instabilities which generally lead to the transition to turbulence. When the flow develops over a curved boundary this transition may occur through the development of the so-called Görtler instability ${ }^{1-5}$, which results from a local unbalance between the centrifugal force and the normal pressure gradient. The boundary layer then exhibits unstable flow motion in the form of pairs of counter-rotating streamwise vortices that gives rise to longitudinal streaks, leading to strongly distorted velocity profiles and normal-to-wall flow motion correlated with local low and high momentum fluxes. Due to their direct application in industrial ${ }^{6,7}$ and geophysical ${ }^{8-10}$ contexts, experimental ${ }^{11-13}$ and numerical ${ }^{14-16}$ studies on the development of the Görtler instability in boundary layer flows were performed using both wavelength-forced and wavelength-free inlet conditions. Inflow conditions showed to be a crucial choice since they lead either to a unique prescribed wavelength of the Görtler instability or to a freely developing system of multiple wavelengths.

For boundary layers developing over a flat plate, previous studies ${ }^{17,18}$, highlight the importance of the inlet conditions in determining the laminar-turbulent transition, not only in terms of the transition Reynolds number $\operatorname{Re}_{\theta, t}$ based on the momentum thickness $\theta$ and the free stream velocity $U_{\infty}$, but also influencing the formation of characteristic flow coherent structures that develop in the boundary layer. This, in turn, can strongly change the behaviour of flow properties such as the drag coefficient with important consequences in industrial and geophysical contexts. For example, Schlatter and Örlü ${ }^{19}$ showed that the skin friction coefficient $C_{f}$ have very different developments depending on the tripping method used to force transition to turbulence. In general, it was observed that, for a Blasius boundary layer over a flat plate, the smaller the transition Reynolds number $R e_{\theta, t}$, the more likely an overshoot of $C_{f}$ was reported depassing the theoretical predictions for a turbulent boundary layer; in contrast, when $\operatorname{Re}_{\theta, t}$ was large $(>2,500)$ a smooth increase of $C_{f}$ from laminar to turbulent predictions over a flat plate has been observed (see also Méndez et al. ${ }^{20}$ ).

In a Görtler flow, the typical evolution of the flow includes a first linear region characterized by a distortion of the mean flow which is well predicted by linear stability theory ${ }^{3}$, followed by a non-linear region characterized by the above-mentioned longitudinal Görtler structures with a mushroom-like spanwise section and upwash/downwash location. Swearingen and Blackwelder ${ }^{12}$ (see also Park and Huerre ${ }^{21}$ ) concluded that the Görtler vortices do not degenerate directly to 
turbulence, instead they set-up a localized shear-layer instability that results in the appearance of secondary instabilities. Their main finding was the association of the secondary instabilities with the high shears in the spanwise direction rather than with those in the wall-normal direction (see also Schultz and Volino ${ }^{22}$ ).

Méndez et al. ${ }^{20}$ studied the laminar-to-turbulent transition in a Blasius flow over a concave wall by forcing the wavelength of the Görtler instability using wall-roughness elements, following the study of Schrader, Brandt, and Zaki ${ }^{14}$, while increasing the spanwise dimension of the computational domain to assure a better transition to turbulence. They identified and characterized four regions encountered in the transition process (linear, nonlinear, transition to turbulence and fully turbulent) and performed a parametric study showing that the transition point is delayed when the radius of curvature is increased, however, at the same critical Görtler number.

So far, eighter numerical (Schrader, Brandt, and Zaki ${ }^{14}$, Méndez et al. ${ }^{20}$ and references herein) or experimental $^{8,9,11-13,22}$ studies focusing on the evolution of the boundary layer over a curved boundary reported the onset of only one unstable Görtler wavelength within the full considered domain. Generally, these numerical studies started with initial laminar flow conditions with the subsequent onset of one Görtler wavelength as predicted from LSA and that degenerated into a turbulent flow. The experiments also include studies were the flow already attained turbulent conditions when reaching the curved boundary. Tani ${ }^{11}$ demonstrated experimentally that Görtler vortices can exist also in a turbulent boundary layer (see also Hopfinger et $^{\text {al }}{ }^{8}$, Albayrak, Hopfinger, and Lemmin ${ }^{9}$, Swearingen and Blackwelder ${ }^{12}$ ). In this cases, the dominant wavelength of the Görtler instability can be predicted using LSA, but a 'turbulent' Görtler number based on the turbulent viscosity $v_{t}$ has to be considered instead of the classical Görtler number defined using the kinematic viscosity $v$.

Here, we perform well resolved Large-Eddy Simulations (LES) of a Blasius boundary layer over a concave wall. We let the Görtler instability develop freely, starting from a linear region and reaching turbulent conditions at the end of the computational domain. In particular, the Reynolds and Görtler numbers at the inlet are set to much higher values $\left(\operatorname{Re}_{\theta_{0}}=1,175, G_{\theta_{0}}=75\right)$ as compared to previous studies. In their study of boundary layer data over a flat plate, Örlü and Schlat$\operatorname{ter}^{23}$ demonstrated that starting with a high laminar $\operatorname{Re}_{\theta}(=750)$ yields different results not only in terms of the skin friction coefficient, but also for integral and global quantities. Here, the imposed high $G_{\theta_{0}}=75$ enables reaching high enough turbulent Görtler numbers in the turbulent region to ensure the onset of a second dominant wavelength. The large spanwise domain permits 


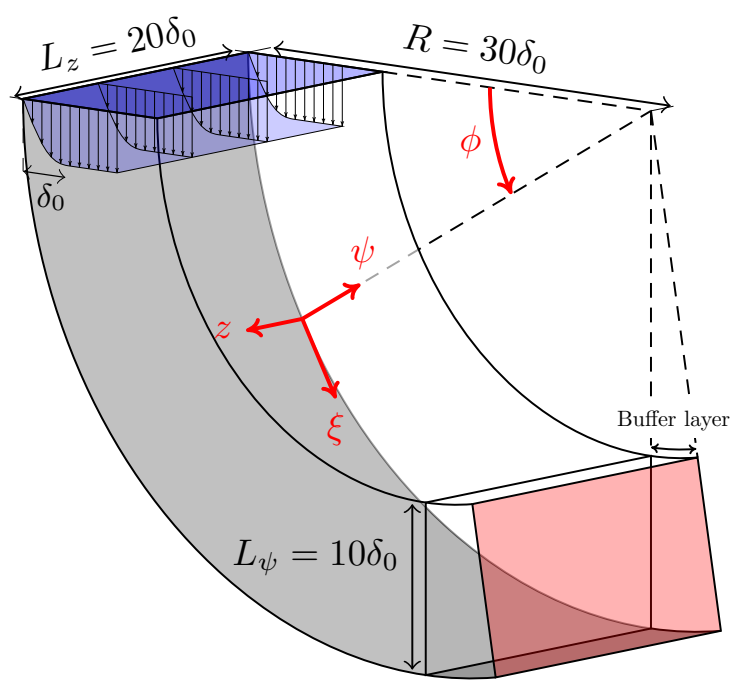

FIG. 1. Sketch of the computational domain. The inlet and outlet are highlighted in blue and red respectively. All dimensions are expressed in terms of the initial boundary layer thickness $\delta_{0}$. The domain for physical analysis spans from $\phi=0$ to $\phi=\pi / 2$, and a buffer layer is used for 10 more degrees.

the presence of up to 52 Görtler vortices in the laminar region, with the observed wavelength corresponding to the theoretical most amplified wavelength predicted by LSA for the present conditions. The skin friction coefficient is found to be accordingly heterogeneous, both in the laminar and turbulent regions with values that exceeds the predictions for a turbulent boundary layer over a flat plate in the turbulent region, similarly to Örlü and Schlatter ${ }^{23}$.

The paper is organized as follows: in section II we describe the numerical resolution method. Section III includes results for the flow topology and the characteristic statistics of each developed region ( $\S$ III A), the comparison with theoretical predictions of linear stability theory $(\S I I I B)$ and the effects on the skin friction coefficient (§III C). Conclusions are given in section IV.

\section{NUMERICAL METHOD}

Large-Eddy Simulations (LES) have been performed using the YALES2 flow solver ${ }^{24}$, which solves the incompressible Navier-Stokes equations for turbulent flows on unstructured meshes using a projection method for pressure-velocity coupling ${ }^{25}$. It relies on fourth-order central finitevolume schemes and on highly efficient linear solvers ${ }^{26}$. The time integration is explicit for convective terms using a fourth-order modified Runge-Kutta scheme, with a semi-implicit integration for the diffusive terms. We used unstructured grids composed by prisms and tetrahedron elements, 


$$
\operatorname{Re}_{\theta_{0}} \quad G_{\theta_{0}} \quad\left(\frac{R}{\theta_{0}}\right)^{1 / 2} \quad \text { Transition method } \quad \Delta \xi^{+} \times \Delta \psi^{+} \times \Delta z^{+}
$$

\begin{tabular}{|c|c|c|c|c|c|}
\hline Méndez et al. ${ }^{20}$ & 75 & 1.5 & 50.0 & Roughness elements & $19.10 \times 0.55 \times 5.80$ \\
\hline Schrader, Brandt, and Zaki ${ }^{14}$ & 75 & 1.5 & 50.0 & Roughness elements & $15.70 \times 0.70 \times 7.10$ \\
\hline Schultz and Volino 22 & 133 & 5.3 & 25.0 & Free-stream turbulence & - \\
\hline Present study & 210 & 13.4 & 16.0 & Numerical noise & $4.90 \times 0.17 \times 4.90$ \\
\hline Present study & 290 & 18.4 & 16.0 & Numerical noise & $4.90 \times 0.17 \times 4.90$ \\
\hline Present study & 1175 & 75.0 & 16.0 & Numerical noise & $4.90 \times 0.17 \times 4.90$ \\
\hline Örlü and Schlatter ${ }^{23}$ & 55 & $\infty$ & 0.0 & Tripping at $R e_{\theta}=55$ & $25.30 \times 14.2 \times 10.8$ \\
\hline Örlü and Schlatter ${ }^{23}$ & 750 & $\infty$ & 0.0 & Tripping at $R e_{\theta}=750$ & $25.30 \times 14.2 \times 10.8$ \\
\hline
\end{tabular}

TABLE I. Physical configuration and numerical resolution. References are sorted by $G_{\theta_{0}}$ in ascending order.

allowing to have a high resolution of the near-wall region. The dynamic Smagorinsky subgridscale model is used ${ }^{27}$.

A sketch of the computational domain is given in figure 1. A local frame $(\xi, \psi, z)$ is used to conveniently characterize the flow in the present curved domain, where $\xi, \psi$ and $z$ are the streamwise, wall-normal and spanwise directions, respectively. The inlet velocity is set to a Blasius boundary layer with a thickness $\delta_{0}$ and a free-stream velocity $U_{\infty}$. The geometry of the computational domain is a 90-degree concave channel with constant curvature $R=30 \delta_{0}$, with a spanwise length $L_{z}=20 \delta_{0}$ and a wall-normal length $L_{\psi}=10 \delta_{0}$. Close to the wall, the mesh size in the streamwise, wall-normal and spanwise directions is $\Delta \xi=3.6 \times 10^{-2} \delta_{0}, \Delta \psi=1.2 \times 10^{-3} \delta_{0}$ and $\Delta z=3.6 \times 10^{-2} \delta_{0}$ respectively, for a total number of mesh cells $N_{c}=2.4 \times 10^{8}$. This ensures that the mesh size at the wall, expressed in wall unit ${ }^{+}$(normalization with the wall friction velocity $u_{\tau}$, and the kinematic viscosity $\left.v\right)$, are $\left(\Delta \xi^{+}, \Delta \psi^{+}, \Delta z^{+}\right)=(4.90,0.17,4.90)$ at the inlet and reach maximum values of $\left(\Delta \xi^{+}, \Delta \psi^{+}, \Delta z^{+}\right)=(28.20,0.98,28.20)$ in the turbulent region. At the bottom and top boundaries (outer and inner radii boundaries), no-slip and free-slip boundary conditions are prescribed, respectively. The flow is set to be periodic in the spanwise direction. At the end of the 90-degree domain, a buffer layer is used $\left(90^{\circ}<\phi<100^{\circ}\right)$ and a convective outflow ${ }^{28}$ after $\left(\phi=100^{\circ}\right)$.

We let Görtler vortices develop spontaneously without forcing the spanwise wavelength, with- 


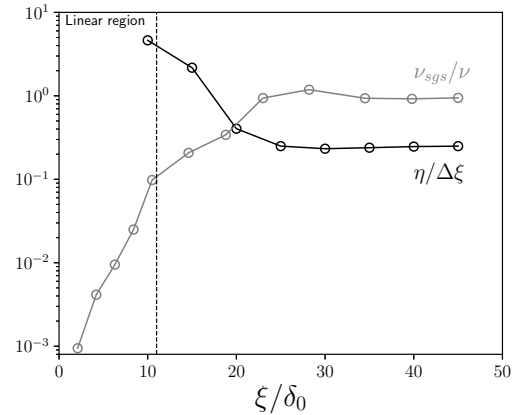

(a)

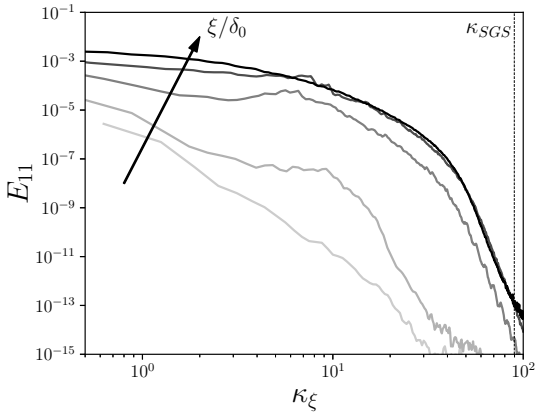

(b)

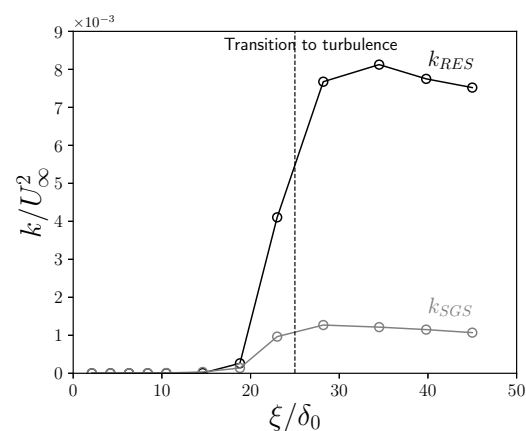

(c)

FIG. 2. (a) Ratio between the Kolmogorov scale $\eta$ and the mesh size in the streamwise direction $\Delta \xi$ (black line), along with the ratio between the sub-grid viscosity $v_{S G S}$ and the kinematic viscosity of the fluid $v$. (b) Streamwise evolution of the energy spectrum $E_{11}$ for $\xi / \delta_{0}=10,15,20,25,35$ (from light grey to black). (c) Streamwise evolution of the explicitly resolved TKE $k_{R E S}$ and the sub-grid TKE $k_{S G S}$.

out free-stream turbulence level nor white noise perturbations. Hence, the centrifugal instability is triggered by numerical noise. The spanwise length of the computational domain was chosen in order to get up to 52 Görtler rolls within the initial linear region, based on linear stability predictions where the expected non-dimensional wavelength is $\Lambda=\left(U_{\infty} \lambda / v\right)(\lambda / R)^{1 / 2}=500$, where $\lambda$ is the wavelength defined as the distance between two pairs of counter-rotating Görtler vortices. This allows to reach converged spatial statistics in the spanwise direction in both the linear and turbulent regions. The Reynolds number based on the inflow boundary layer thickness $\delta_{0}$ is $R e_{\delta_{0}}=\left(U_{\infty} \delta_{0}\right) / v=10,000$ while the Reynolds number based on the inflow momentum thickness is $\operatorname{Re}_{\theta_{0}}=\left(U_{\infty} \theta_{0}\right) / v=1,175$. The Görtler number is consequently $G_{\theta_{0}}=\operatorname{Re}_{\theta_{0}}\left(\theta_{0} / R\right)^{1 / 2}=75$.

\section{A. Numerical validation and turbulent state}

Figure 2a displays the streamwise evolution of the Kolmogorov scale $\eta=\left(v^{3} / \varepsilon\right)^{1 / 4}$ (explicitly determined using structure functions ${ }^{29}$ ) normalized by the longitudinal mesh size $\Delta \xi$ (black line) and the evolution of the sub-grid eddy viscosity $v_{s g s}$ normalized by the kinematic viscosity $v$ (grey line). We see that $v_{S G S} / \nu \ll 1$ and $\eta / \Delta \xi \approx 5$ in the linear region, so that the chosen grid size at the wall in the linear region $\left(\xi / \delta_{0} \leq 11\right)$ is sufficiently small to resolve all scales and the LES is practically equivalent to a DNS.

Figure $2 \mathrm{~b}$ gives the energy spectrum as a function of the streamwise wavenumber $\kappa_{\xi}$ at different streamwise positions (different solid lines), showing that starting from $\xi / \delta_{0}=10$ all energetic 


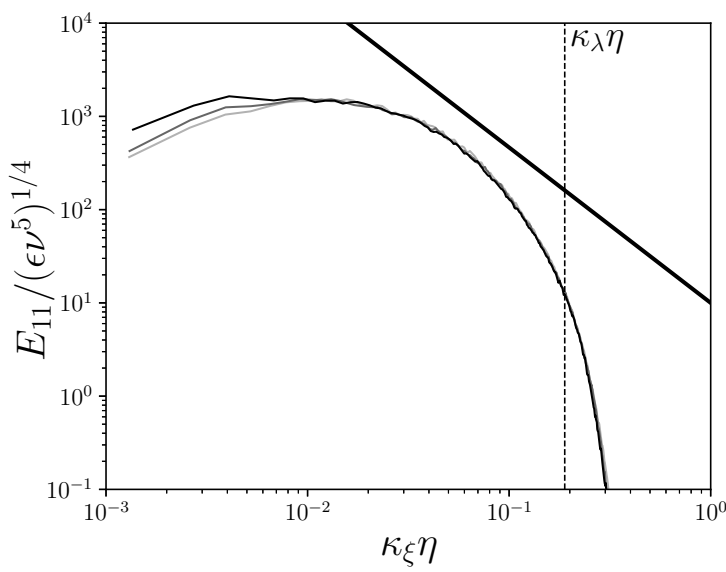

(a)

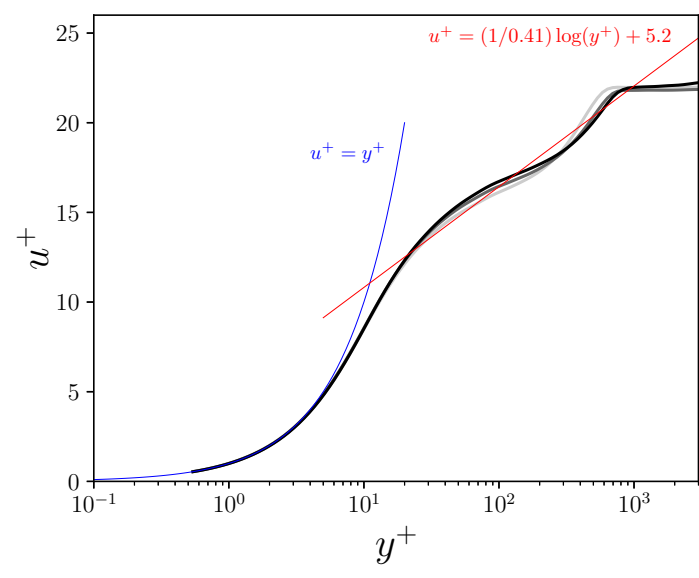

(b)

FIG. 3. (a) Normalized energy spectrum for $\xi / \delta_{0}=35,40,45$ (from light grey to black solid lines), with the $-5 / 3$ slope (black solid line) and the Taylor micro-scale (vertical dotted line). (b) Velocity profiles in wall units $u^{+}=f\left(y^{+}\right)$for $\xi / \delta_{0}=35,40,45$ (from light grey to black circles).

scales are resolved. Further downstream, we see the evolution of the spectra consistent with an energy transfer from small to larger wavenumbers up to the cut-off wavenumber $\kappa_{S G S}$ (see vertical dotted line in figure $2 b$ ) representative of the sub-grid model. Figure $2 \mathrm{c}$ shows the streamwise evolution of the normalized directly resolved $\left(k_{R E S}\right)$ and modeled $\left(k_{S G S}\right)$ turbulent kinetic energy (TKE). After transition to turbulence, $k_{R E S}$ accounts for less than $20 \%$ of the total TKE, which gives a criterion for good LES resolution ${ }^{29,30}$.

To assess the turbulence level reached in the last part of the computational domain we plot in figure $3 \mathrm{a}$ the normalized energy spectrum $E_{11} /\left(\varepsilon v^{5}\right)^{1 / 4}$ as a function of $\kappa_{\xi} / \eta$ for $\xi / \delta_{0}=$ $(35,40,45)$. The spectra converge and the inertial zone (i.e. Taylor microscale $\kappa_{\lambda} \eta$, dashed black line) is partially resolved and consistent with a $-5 / 3$ power law. We also estimated the Reynolds number $\operatorname{Re}_{\lambda}=u^{\prime} \lambda / v$ based on the Taylor microscale $\lambda=\left(v u^{\prime^{2}} / \varepsilon\right)^{1 / 2}$ and found values $\operatorname{Re} \lambda>100$ for $\xi / \delta_{0}>15$, with an increasing value up to $R e_{\lambda} \approx 200$ at the end of the computational domain.

The velocity profiles expressed in wall-units $u^{+}=f\left(y^{+}\right)$computed at the end of the domain $\left(\xi / \delta_{0}=(35,40,45)\right)$ are displayed in figure $3 \mathrm{~b}$. They converge toward a turbulent velocity profile in agreement with the $\log \operatorname{law} u^{+}=(1 / 0.41) \log \left(y^{+}\right)+5.2$ found in turbulent boundary layers over flat plates. We note that the velocity profiles exhibit an inflection point, specific of a secondary motion in the flow induced by the presence of Görtler vortices.

An overall assessment of the boundary layer development can be obtained through the boundary 


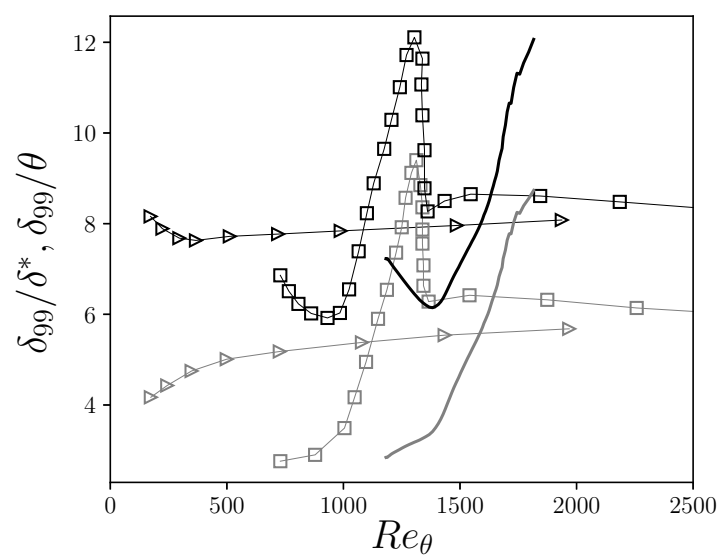

(a)

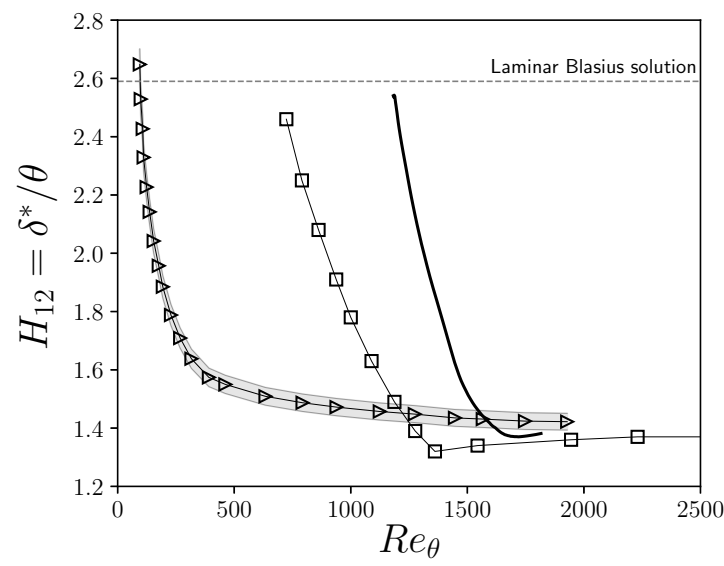

(b)

FIG. 4. (a) Evolution of the boundary layer thickness $\delta_{99}$ normalized by the displacement thickness $\delta^{*}$ (grey) and the momentum thickness $\theta$ (black) versus $R e_{\theta}$. Present results with $R_{\theta_{0}}=1175$ (solid lines) are compared with results from Örlü and Schlatter ${ }^{23}$ (squares: $\operatorname{Re}_{\theta_{0}}=750$; triangles: $\operatorname{Re}_{\theta_{0}}=55$ ). (b) Shape factor as a function of $R e_{\theta}$. A $2 \%$ error with respect to results from Örlü and Schlatter ${ }^{23}$ at $R e_{\theta_{0}}=55$ is highlighted in grey.

layer thickness $\delta_{99}$ as well as its integral measures, i.e. the displacement $\left(\delta^{*}\right)$ and momentum-loss $(\theta)$ thicknesses. The ratio between $\delta_{99}$ and $\delta^{*}$ (grey solid line) and $\theta$ (black solid line) is depicted in figure $4 \mathrm{a}$, along with the the shape factor $\left(H_{12}=\delta^{*} / \theta\right)$ in $4 \mathrm{~b}$, as a function of $\operatorname{Re}_{\theta}$. We compare our results to those of Örlü and Schlatter ${ }^{23}$ for two cases: the first having $R e_{\theta_{0}}=55$ (triangles) and the second with $R e_{\theta_{0}}=750$ (squares). As pointed out by Örlü and Schlatter ${ }^{23}$, a strong increase of the normalized boundary layer thicknesses occurs when transitioning to turbulence if the initial Reynolds number is large $\left(R e_{\theta_{0}}=750\right.$ for Örlü and Schlatter ${ }^{23}$ and $R e_{\theta_{0}}=1,175$ in the present study), which clearly contrasts with the smooth increase in the case of $\operatorname{Re}_{\theta_{0}}=55$. The shape factor $H_{12}$ reveals a similar behaviour as in the case of Örlü and Schlatter ${ }^{23}$ with a turbulent state reached at the end of the computational domain however with values that are $8 \%$ of the convergence limit (cf. Örlï and Schlatter ${ }^{23}$ ). These results confirm thus the findings of Örlü and Schlatter ${ }^{23}$ for which starting with a higher laminar $\operatorname{Re}_{\theta, 0}$ retards reaching the fully turbulent regime. 


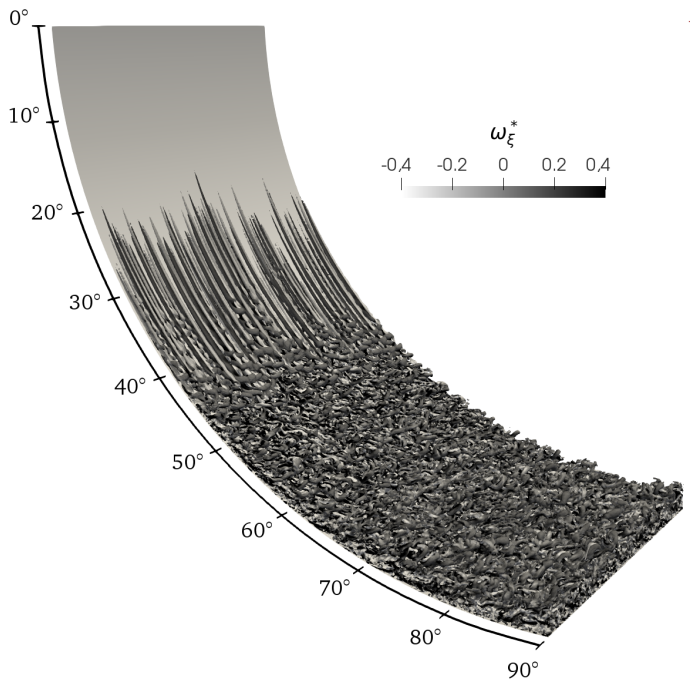

(a)

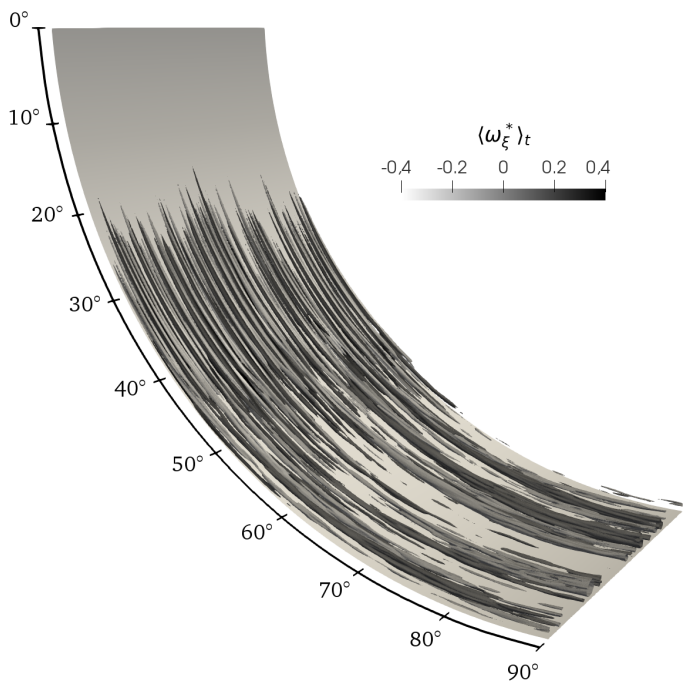

(b)

FIG. 5. Three-dimensional views of iso-contours of the normalized $Q$-criterion $Q^{*}=Q R^{2} / U_{\infty}^{2}=1$, computed from the instantaneous flow velocity field (a) and the mean flow velocity field (b) colored by the instantaneous non-dimensional vorticity $\omega_{\xi}^{*}=\omega_{\xi} \delta_{0} / U_{\infty}$ and the mean non-dimensional streamwise vorticity $\left\langle\omega_{\xi}^{*}\right\rangle_{t}=\left\langle\omega_{\xi}\right\rangle_{t} \delta_{0} / U_{\infty}$. Streamwise streaks structures (Görtler vortices) are visible in the non-linear region of the instability $\left(22^{\circ}<\phi<42^{\circ}\right)$, and persistent in the turbulent region (b).

\section{RESULTS}

In the following, ensemble average $\langle\cdot\rangle$ is performed along homogeneous samples. Averaging over time at each point $(\xi, \psi, z)$ provides e.g. temporal mean velocity $\langle\mathbf{u}\rangle_{t}(\xi, \psi, z)$, where $\mathbf{u}=(u, v, w)$ is the vector of the three velocity components in the longitudinal, wall-normal and spanwise directions respectively. Additional averaging along the spanwise direction of the domain provides temporal and spatial mean velocities, e.g. $\langle\mathbf{u}\rangle_{t, z}(\xi, \psi)$.

\section{A. Flow topology}

The topology of the flow is described using a non-dimensional $Q$-criterion defined as $Q^{*}=$ $\left(\Omega_{i j} \Omega_{i j}-S_{i j} S_{i j}\right) /\left(2 \delta_{0}^{2} / U_{\infty}^{2}\right)$. Herein, $\Omega_{i j}$ is the vorticity tensor and $S_{i j}$ the strain rate tensor. A three-dimensional view of the iso-contours of the $Q$-criterion set to $Q^{*}=10^{-3}$ is shown in figure 5. Figure 5(a) is computed from the instantaneous velocity field $\mathbf{u}$ colored by the instantaneous non-dimensional streamwise vorticity $\omega_{\xi}^{*}=\omega_{\xi} \delta_{0} / U_{\infty}$, while figure $5(\mathrm{~b})$ is computed from the 


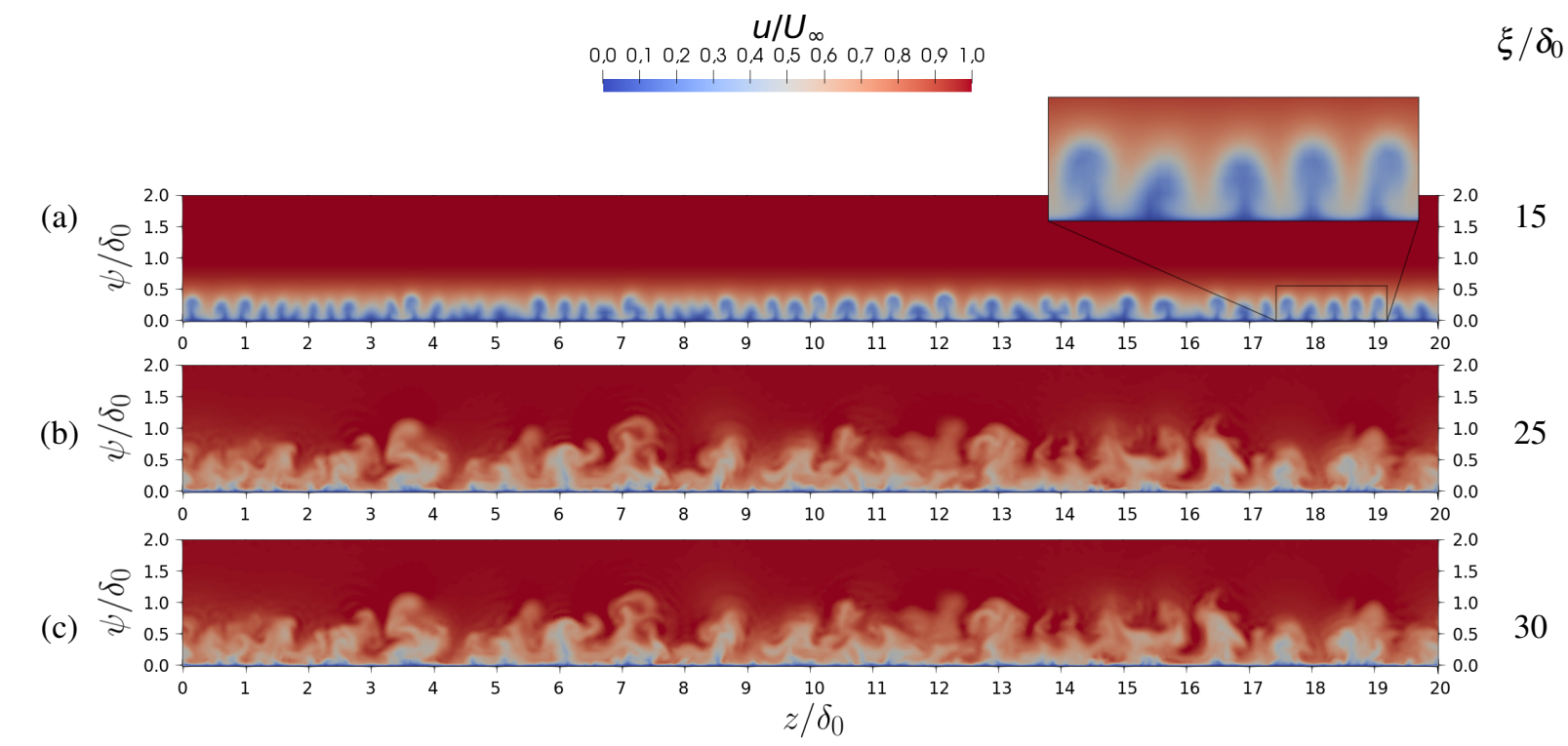

FIG. 6. Instantaneous streamwise velocity field $u / U_{\infty}$ at $\xi / \delta_{0}=15$ (a), 25 (b) and 30 (c). In (a) mushroomlike structures of the Görtler instability are evident (see inset for a zoomed view). For $\xi / \delta_{0}>25$ (b-c), a scale multiplicity is observed in the instantaneous velocity fields (i.e. turbulent state).

mean flow $\langle\mathbf{u}\rangle_{t}$ colored by the mean non-dimensional streamwise vorticity $\left\langle\omega_{\xi}^{*}\right\rangle_{t}=\left\langle\omega_{\xi}\right\rangle_{t} \delta_{0} / U_{\infty}$. The longitudinal streak structures present for $21^{\circ}<\phi<42^{\circ}$ are observed both in figures 5(a) and 5(b) meaning that they are spatially steady, which is characteristic for Görtler vortices. In figure $5(\mathrm{a})$, we see that the onset of the Görtler vortices appears at about $\xi / \delta_{0}=11\left(\phi=21^{\circ}, \operatorname{Re}_{\theta}=\right.$ $1,200)$, and the transition to turbulence starts at about $\xi / \delta_{0}=22\left(\phi=42^{\circ}, \operatorname{Re}_{\theta}=1350\right)$. The turbulent region is reached at $\xi / \delta_{0}>31\left(\phi>59^{\circ}, \operatorname{Re}_{\theta}=1,500\right)$ (see also §II A). The iso-contour of the $Q$-criterion computed from the mean flow velocity field (figure $5 \mathrm{~b}$ ) exhibits large scale longitudinal vortical Görtler structures in both the transition and in the turbulent region. Spanwise instantaneous streamwise velocity fields are displayed in figure 6 at three different streamwise locations $\xi / \delta_{0}=(15,25,30)$ (a,b,c, respectively) highlighting the typical mushroom like structure of the Görtler instability ${ }^{13}$, well defined in the initial development region (figure 6a) while figure $6(b, c)$ shows the onset of scale multiplicity further downstream.

Since the Görtler vortices are spatially steady, they induce a distortion of the mean flow ${ }^{5}$ which leads to a spanwise heterogenity of the flow properties. A measure of the spanwise heterogeneity level (SHL) of the mean flow has been proposed by Méndez et al. ${ }^{20}$ as follows:

$$
\mathbf{u}_{S H L}(\xi)=\sqrt{\frac{1}{L_{z}} \int_{0}^{L_{z}}\left[\langle\mathbf{u}\rangle_{t}^{\prime}(\xi, \psi, z)\right]^{2} \mathrm{~d} z}
$$


where $\langle\mathbf{u}\rangle_{t}^{\prime}(\xi, \psi, z)=\langle\mathbf{u}\rangle_{t}(\xi, \psi, z)-\langle\mathbf{u}\rangle_{t, z}(\xi, \psi)$ is the spanwise fluctuation of the mean velocity field $\langle\mathbf{u}\rangle_{t}(\xi, \psi, z)$.

Equation (1) represents the spanwise standard deviation of the temporal mean flow, and the streamwise evolution of its value at the wall-normal position $\psi=0.25 \delta_{0}$ is displayed in figure 7(a). We see that all three components of $\mathbf{u}_{S H L}$ rapidly increase in the first region which corresponds to the linear growth of the Görtler instability and starts to saturate at $\xi / \delta_{0} \approx 11$. The level of heterogeneity is maintained for $\xi / \delta_{0}>25$ for all three components. This is not observed in a turbulent boundary layer over a flat plate, where a statistical spanwise homogeneity of the flow is expected.

Selected profiles of $\mathbf{u}_{S H L}$ are shown in figure 7(b) along with the associated mean streamwise velocity profiles $\langle u\rangle_{t, z}$. At $\xi / \delta_{0}=10$ (linear region), the computed $\langle u\rangle_{t, z}$ matches the Blasius solution. $u_{S H L}$ and $v_{S H L}$ have maximum values for $\psi / \delta_{0}=0.12$ and $\psi / \delta_{0}=0.16$, respectively while $w_{S H L}$ has two maxima reached for $\psi / \delta_{0}=0.05$ and $\psi / \delta_{0}=0.28$. Note that the wall-normal positions of the maxima of all three components of $\mathbf{u}_{S H L}$ are consistent with the observation of the Görtler vortices (cf. figure 6a). At $\xi / \delta_{0}=25$ (non-linear region), an inflection point is observed on the mean streamwise velocity profile $\langle u\rangle_{t, z}$ (grey line in figure $7 \mathrm{~b}$ ), which is due to the development of the Görtler vortices. Wall-normal profiles of $u_{S H L}$ and $w_{S H L}$ are strongly influenced by the nonlinear effects and the wavelength multiplicity thus having complex shapes while the $v_{S H L}$ profile shape remains nearly the same. The width of all three profiles is thickening in the non-linear region, which might be associated with a sudden increase of turbulent state (see the appearance of three-dimensional structures in figure $5 \mathrm{a}$ and non-linear saturation in figure $7 \mathrm{a}$ ). At $\xi / \delta_{0}=40$ in the turbulent region, the mean velocity profile as well as $\mathbf{u}_{S H L}$ profiles are thicker, and an inflection point is still observed on the $\langle u\rangle_{t, z}$ profile, consistent with the large-scale streamwise vortices observed in the turbulent region (figure 5c). In this region, $u_{S H L}$ and $w_{S H L}$ have their maximum value very close to the wall at $\psi / \delta_{0}=0.08$, while $v_{S H L}$ still keeps the same profile shape and its maximum moves away from the wall, similarly to what is observed when Görtler vortices grow in the non-linear region.

In order to extract the wavelengths responsible for the spatial heterogeneity, we focus on the wall-normal component of the velocity, which is associated with the upwash and downwash motions, characteristic of the Görtler instability. A spatial FFT is therefore performed in the spanwise direction on the velocity fluctuation $\langle v\rangle_{t}^{\prime}$ and the streamwise evolution of the power spectral density is shown in figure 8(a). Unlike the previous studies of Méndez et al. ${ }^{20}$ and Schrader, Brandt, and 


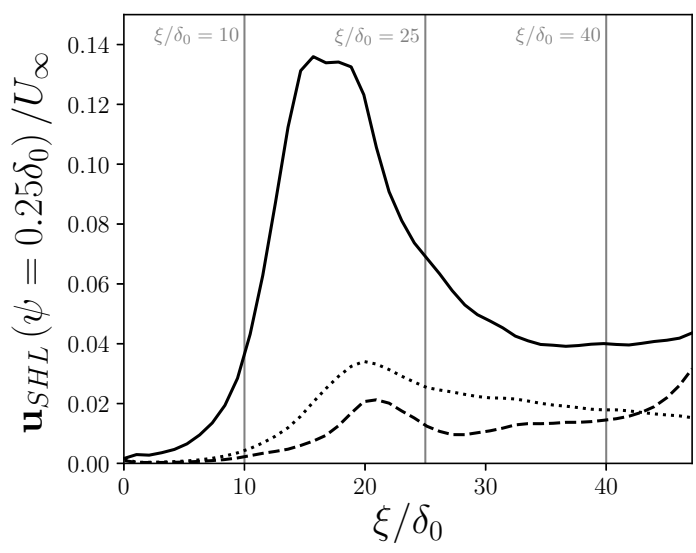

(a)

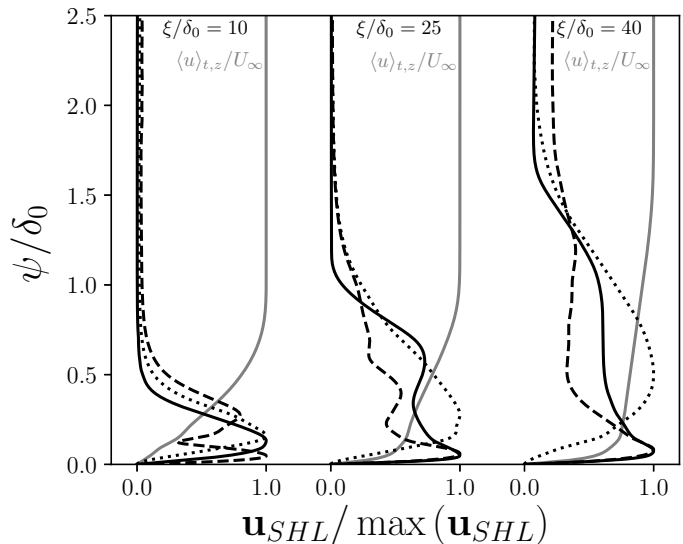

(b)

FIG. 7. (a) Streamwise evolution of $\mathbf{u}_{S H L}$ at $\psi=0.25 \delta_{0}$ (Eq. 1) for the longitudinal (solid line), wall-normal (dashed line), and spanwise (dotted line) velocity components with the saturation reached at $\xi / \delta_{0} \approx 22$. (b) Selected vertical profiles at $\xi / \delta_{0}=(10,25,40)$ (from left to right, respectively) for $\mathbf{u}_{S H L}$ and normalized by their maximum. The grey solid lines in (b) represent the base state Blasius profile.

Zaki ${ }^{14}$ where the instability wavelength is forced, here we report the onset of various wavelengths, also observed in Tandiono, Winoto, and Shah ${ }^{13}$.

In the linear region $\left(2<\xi / \delta_{0}<11\right)$, multiple wavelengths can be identified (cf. figure $8 \mathrm{a}$ ), and the most amplified wavelength is found to be $\lambda_{1}=0.385 \delta_{0} . \lambda_{1}$ is dominant for $2<\xi / \delta_{0}<22$ which corresponds to both linear and non-linear regions. At the breakdown of the Görtler vortices (figure 7a), larger wavelengths $\left(0.5 \delta_{0}<\lambda<1.0 \delta_{0}\right)$ are found in the spectrum which persist only for a distance $\Delta \xi \approx 6 \delta_{0}$.

Further downstream in the turbulent region $\left(\xi / \delta_{0}>30\right)$, the dominant wavelength in the flow is found to be $\lambda_{2}=1.55 \delta_{0}$.

\section{B. Comparison to linear stability theory}

In order to estimate the theoretical predicted growth rates and most unstable wavelengths, we extended the LSA of Floryan and Saric ${ }^{3}$ for a larger domain of the non-dimensional wavenumber $0.03<\alpha \theta<3.0$, with $\alpha \theta=(2 \pi / \lambda) \theta$, and for Görtler numbers $0.5<G_{\theta}<100.0$. For this, we considered the linear perturbation equations of continuity and momentum for a Blasius boundary layer over a curved wall using a normal modes approach. The governing stability equations read 
Linear to turbulent Görtler instability transition

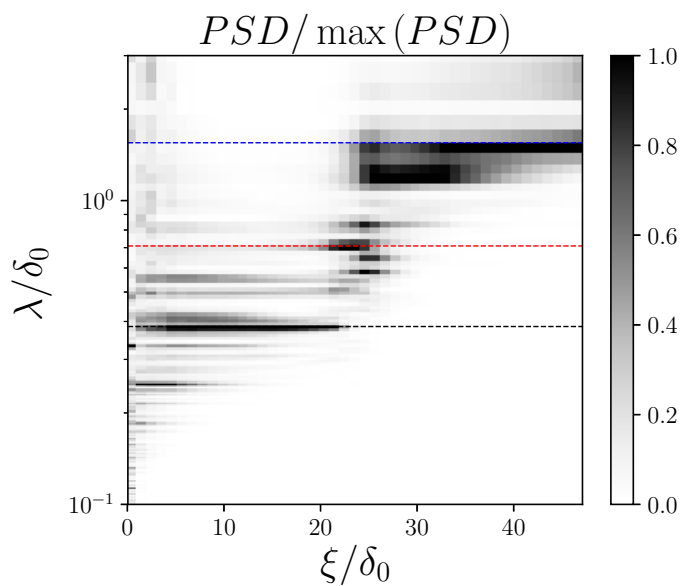

(a)

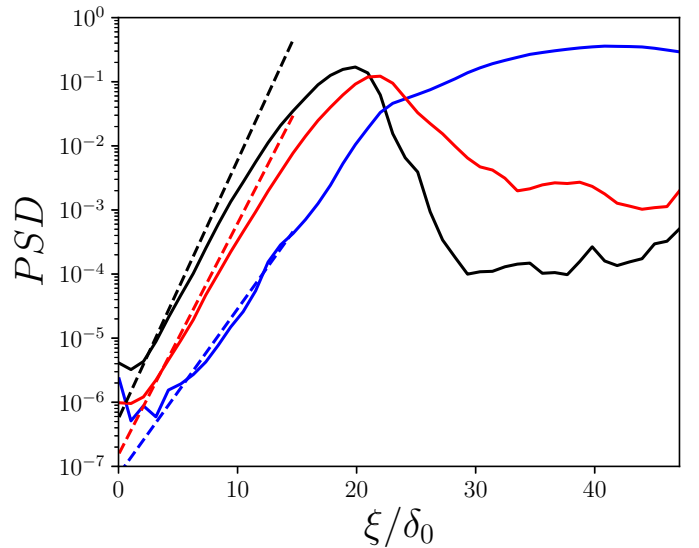

(b)

FIG. 8. (a) Spectrogram from a spatial FFT in the spanwise direction on the wall-normal disturbance velocity $\langle v\rangle_{t}^{\prime}$ normalized by the local maximum amplitude. Two dominant wavelengths emerge in the different development regions: $\lambda_{1}=0.385 \delta_{0}$ (linear region, black dotted line), $\lambda_{2}=1.55 \delta_{0}$ (turbulent region, blue dotted line) and an intermediate wavelength $\lambda=0.709 \delta_{0}$ (red dotted line); (b) respective streamwise evolution of the power spectral densities (solid lines) with the theoretical growth from LSA (dashed lines).

then

$$
\begin{gathered}
\beta \hat{u}+\frac{\mathrm{d} \hat{v}}{\mathrm{~d} \psi}+\alpha \hat{w}=0 \\
\hat{u} \frac{\partial U}{\partial \xi}+\beta U \hat{u}+\hat{v} \frac{\partial U}{\partial \psi}+V \frac{\mathrm{d} \hat{u}}{\mathrm{~d} \psi}-\frac{\mathrm{d}^{2} \hat{u}}{\mathrm{~d} \psi^{2}}+\alpha^{2} \hat{u}=0 \\
\beta U \hat{v}+\hat{u} \frac{\partial V}{\partial \xi}+\hat{v} \frac{\partial V}{\partial \psi}+V \frac{\mathrm{d} \hat{v}}{\mathrm{~d} \psi}+2 G^{2} U \hat{u}+\frac{\mathrm{d} \hat{p}}{\mathrm{~d} \psi}-\frac{\mathrm{d}^{2} \hat{v}}{\mathrm{~d} \psi^{2}}+\alpha^{2} \hat{v}=0 \\
\beta U \hat{w}+V \frac{\mathrm{d} \hat{w}}{\mathrm{~d} \psi}-\alpha \hat{p}-\frac{\mathrm{d}^{2} \hat{w}}{\mathrm{~d} \psi^{2}}+\alpha^{2} \hat{w}=0
\end{gathered}
$$

where the perturbation velocities are defined as

$$
\begin{gathered}
{\left[u^{\prime}, v^{\prime}, p^{\prime}\right]=[\hat{u}(\psi), \hat{v}(\psi), \hat{p}(\psi)] \cos (\alpha z) \exp (\beta \xi)} \\
w^{\prime}=\hat{w}(\psi) \sin (\alpha z) \exp (\beta \xi)
\end{gathered}
$$

with $(U, V)$ the base flow, $\alpha$ the wavenumber, $\beta$ the spatial growth rate and $(\hat{u}, \hat{v}, \hat{w})$ the spatial modes.

The system of equations (2-5) is expressed in matrix form through $M_{i j}$

$$
\frac{\mathrm{d} \zeta_{i}}{\mathrm{~d} \psi}=\mathrm{M}_{i j}(\psi) \zeta_{j}
$$


where $\zeta_{1}=\hat{u}, \zeta_{2}=\frac{\mathrm{d} \hat{u}}{\mathrm{~d} \psi}, \zeta_{3}=\hat{v}, \zeta_{4}=\frac{\mathrm{d} \hat{v}}{\mathrm{~d} \psi}, \zeta_{5}=\frac{\mathrm{d}^{2} \hat{v}}{\mathrm{~d} \psi^{2}}, \zeta_{6}=\frac{\mathrm{d}^{3} \hat{v}}{\mathrm{~d} \psi^{3}}$, which constitutes an eigenvalue problem with boundary conditions

$$
\begin{gathered}
\zeta_{1}=\zeta_{3}=\zeta_{4}=0 \text { for } \psi=0 \\
\zeta_{1}=\zeta_{3}=\zeta_{4}=0 \text { for } \psi \rightarrow \infty,
\end{gathered}
$$

for which solutions exist only for specific sets of $(\alpha, \beta, G)^{3}$. It is solved as a boundary value problem using a 4th-order Runge-Kutta integration method following the work of Petitjeans ${ }^{31}$. The value of $\beta$ which fulfills the boundary conditions (10) is calculated for a set of given $(G, \alpha)$ values $^{3,31}$.

Figure 8(b) is extracted from figure $8(a)$ for the two dominant wavelengths $\lambda_{1}$ (black line) and $\lambda_{2}$ (blue line) along with an intermediate wavelength of about $2 \lambda_{1}$ (red line). It shows the streamwise evolution of the energy contained in the wall-normal component of the velocity fluctuations for each of the above identified wavelengths. The estimated theoretical growth rates for each of these three wavelengths are reported in figure 8(b) as linear dashed lines, revealing a good agreement between the LSA predictions and the simulation results. The energy associated with $\lambda_{1}$ and $2 \lambda_{1}$ is damped further downstream at $\xi / \delta_{0} \approx 25$, while the energy associated with $\lambda_{2}$ keeps growing, hence becoming the dominant wavelength for $\xi / \delta_{0} \geq 30$ until the end of the computational domain.

We predict the theoretical most amplified wavelength for the Görtler number $G_{\theta_{0}}=75$ and a base flow corresponding to a Blasius boundary layer in the linear region of $\lambda_{1}^{\text {th }}=0.38 \delta_{0}$, which matches the present observation.

As already observed experimentally $8,9,11,12$ the Görtler instability may exist also in a fully developed turbulent boundary layer. Tani ${ }^{11}$ argued that the laminar boundary layer Görtler instability diagram can be used to determine the instability characteristics of a turbulent boundary layer if the kinematic viscosity is replaced by the turbulent viscosity in the definition of the Görtler number. In this case, a turbulent Görtler number is the relevant parameter, defined as $G_{T}=U_{\infty} \theta / v_{t}(\theta / R)^{1 / 2}$. Figure 9(a) (solid line) displays the turbulent Görtler number $G_{T}$, in which the turbulent viscosity is estimated using the same approach of Tani ${ }^{11}$, who assumed a constant turbulent viscosity in the outer part of the boundary layer, for which Clauser ${ }^{32}$ suggested the expression $v_{t}=0.018 U_{\infty} \delta^{\star}$, where $\delta^{\star}$ is the displacement thickness. We also estimated the $G_{T}$ by calculating directly the turbulent viscosity using the Boussinesq hypothesis as 


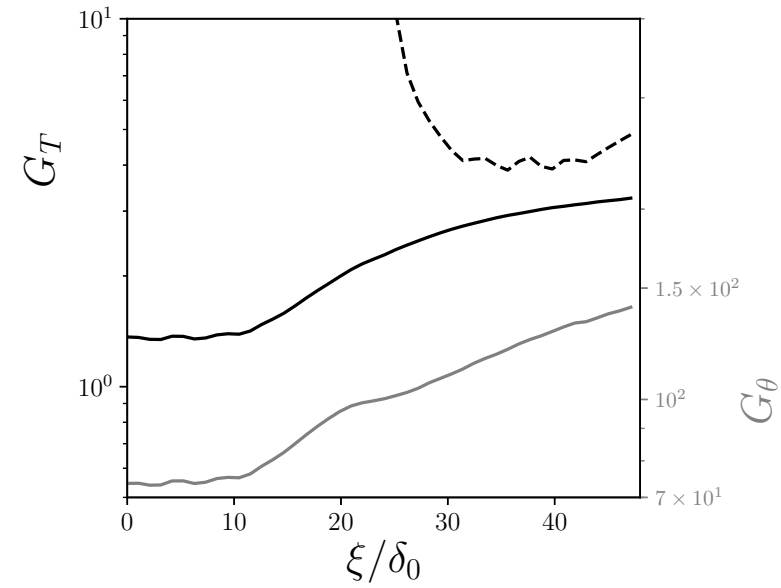

(a)

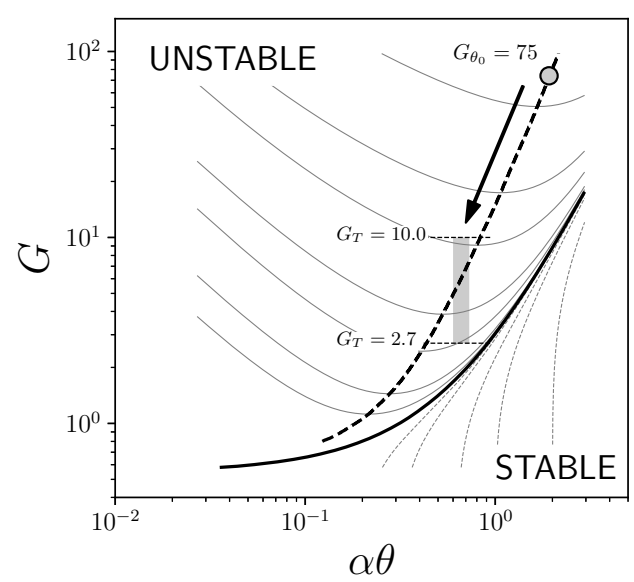

(b)

FIG. 9. (a) Variation of the turbulent Görtler number $G_{T}$ in the longitudinal direction calculated using the turbulent viscosity with the mixing length model $v_{t}=0.018 U_{\infty} \delta^{\star}$ from Clauser $^{32}$ (solid line), where $\delta^{\star}$ is the displacement thickness, and from the Boussinesq hypothesis $v_{t}=-\left\langle u^{\prime} v^{\prime}\right\rangle_{t, z} / \partial_{\psi}\langle u\rangle_{t, z}$ (dashed line). For comparison, $G_{\theta}$ is also plotted (grey line). (b) Curves of constant non-dimensional amplification rates $\beta \theta \operatorname{Re}_{\theta}$. The black thick solid line and thick dashed lines correspond to the stability limit and the curve of maximum amplification rate, respectively. Present LES results are highlighted by the grey box, considering the turbulent Görtler number $G_{T}=U_{\infty} \theta / v_{t}(\theta / R)^{1 / 2}$.

$v_{t}=-\left\langle u^{\prime} v^{\prime}\right\rangle_{t, z} / \partial_{\psi}\langle u\rangle_{t, z}$ (dashed line). Figure 9 includes also the classical Görtler number $G_{\theta}$ based on the kinematic viscosity $v$ (grey line) for comparison. We see that both estimations for $G_{T}$ are very similar, and the turbulent Görtler number ranges within $2.7<G_{T}<10$ in the turbulent region for $\xi>25 \delta_{0}$.

The spatial amplification rate curves obtained from the LSA are displayed in figure 9(b), with the thick black line being the neutral stability curve. The range of the reported turbulent Görtler numbers $G_{T}$ and normalized wavelengths $\alpha \theta$ (note that $\alpha \theta$ varies with $\theta$ since the wavelength $\lambda_{2}$ is kept constant) extracted from the present LES are highlighted within the grey box: the turbulent domain is clearly Görtler unstable. Moreover, the dominant wavelength observed in the present simulation, $\lambda_{2}$, corresponds to a normalized wavenumber $\alpha \theta \approx 0.6$ that fairly fits the dominant wavenumber given by the LSA, represented by the dashed line of maximum amplification rate in figure 9(b). Hence, during transition to turbulence, the effective Görtler number decreases (from $G_{\theta, 0}$ to $G_{T}$ ), favoring the onset of larger wavelengths, in accordance with the theoretical LSA predictions and with the present observations. In previous numerical studies $G_{\theta, 0}$ is smaller (cf. 
table I) so that the turbulent Görtler number falls close or even below the neutral stability curve (cf. figure 9b). Also, at such smaller $G_{T}$ the most unstable modes have smaller wavenumbers so that the formation of Görtler vortices may be inhibited by a limited computational domain in the spanwise direction.

From the spectral analysis, we find that in the linear region, the equivalent number of pairs of counter-rotating vortices corresponding to the most amplified wavelength $\lambda_{1}$ is $N_{\lambda_{1}}=52$, which is sufficiently large to perform a phase-based statistical study in the spanwise direction with converged statistics. Considering that the spanwise flow heterogeneity is mainly driven by the dominant wavelength, the phase-averaged velocity field $\tilde{\mathbf{u}}(\xi, \psi, \tilde{z})$ is defined as:

$$
\langle\tilde{\mathbf{u}}\rangle_{t}(\xi, \psi, \tilde{z})=\frac{1}{N_{\lambda_{\kappa}}} \sum_{i=0}^{N_{\lambda_{\kappa}}-1}\langle\mathbf{u}\rangle_{t}\left(\xi, \psi, \tilde{z}+i \lambda_{\kappa}\right),
$$

where $\tilde{z} \in\left[0, \lambda_{\kappa}\right]$ is the reduced spanwise component, and $\kappa=1,2$ represents the two dominant wavelengths. Similarly, we introduce the phase-averaged fluctuation velocity field $\langle\tilde{\mathbf{u}}\rangle_{t}^{\prime}(\xi, \psi, \tilde{z})=$ $\langle\tilde{\mathbf{u}}\rangle_{t}(\xi, \psi, \tilde{z})-\langle\mathbf{u}\rangle_{t, z}(\xi, \psi)$.

In the linear region, the computed velocity field within $\tilde{z}$ is compared with the theoretical expression of the spatial modes $\hat{\mathbf{u}}$. We expect the present results to fit the expression of the velocity disturbances defined in the LSA:

$$
\langle\tilde{\mathbf{u}}\rangle_{t}^{\prime}(\xi, \psi, \tilde{z})=\hat{\mathbf{u}}(\psi)[\cos (\alpha \tilde{z}), \cos (\alpha \tilde{z}), \sin (\alpha \tilde{z})] \exp (\beta \xi)
$$

In order to compute the spatial mode $\hat{\mathbf{u}}$ using equation (12), we perform a cross-correlation of the fluctuation velocity $\langle\tilde{\mathbf{u}}\rangle_{t}^{\prime}$ and the harmonic functions $[\cos (\alpha \tilde{z}), \cos (\alpha \tilde{z}), \sin (\alpha \tilde{z})]$, where $\alpha$ is obtained using the spanwise FFT. The spatial growthrate $\beta$ is not needed since $\hat{\mathbf{u}}$ is normalized by the maximum value of $\hat{u}$ denoted $\hat{u}_{\max }{ }^{3}$.

The ensemble average profiles at $\xi / \delta_{0}=7$ representative of the linear region are shown in figure 10 (a-c) as solid lines and compared with the obtained LSA solutions (dashed lines). The dispersion of the computed local modes are highlighted with the shaded regions. The agreement is good, with exception of a slight shift between the two curves and an overestimation of $\hat{v}$, more likely due to the simultaneous presence of other wavelengths with similar amplitudes in the simulation profiles, as observed in figure 8(a).

The same statistical analysis is applied to the higher dominant wavelength $\lambda_{2}$, both in the initial linear region $\left(2<\xi<11 \delta_{0}\right)$ and in the turbulent region $\left(\xi>35 \delta_{0}\right)$ (figure $10(\mathrm{~d}-\mathrm{f})$ ). In the linear region, spatial modes associated with $\lambda_{2}$ obtained from the simulation compare well with the 


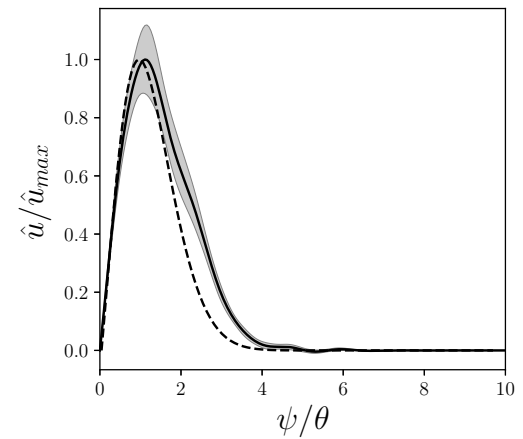

(a)

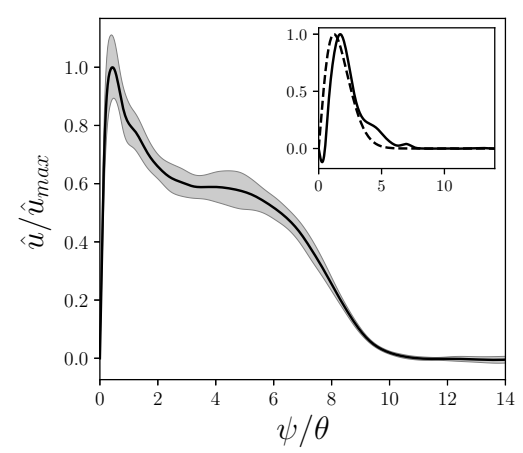

(d)

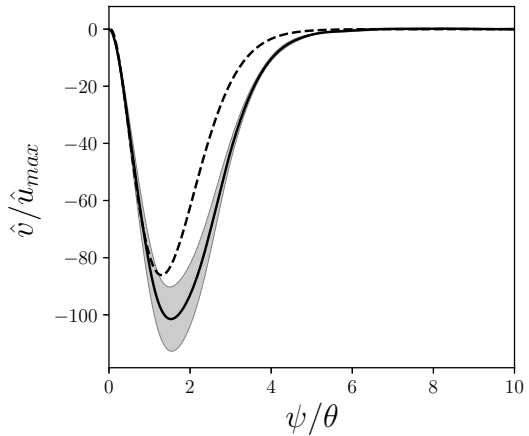

(b)

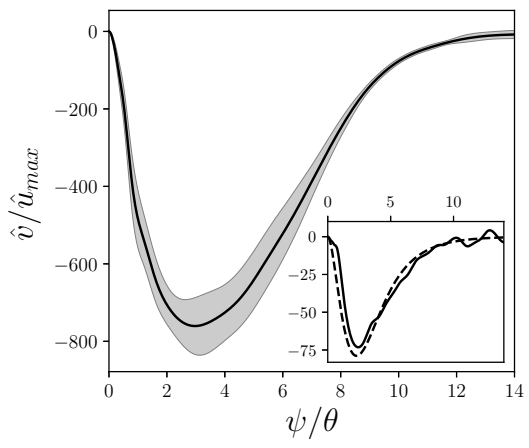

(e)

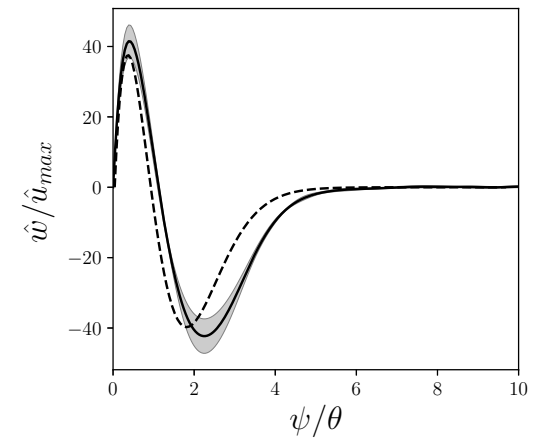

(c)

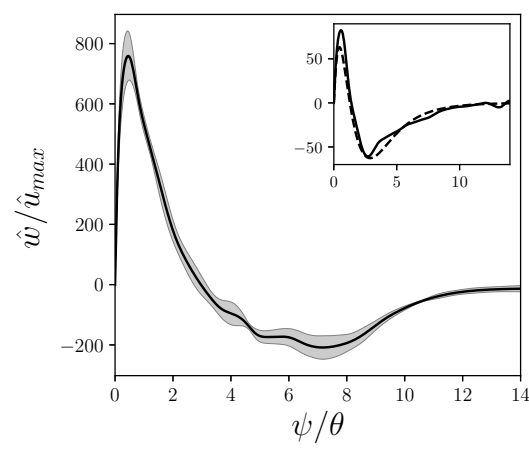

(f)

FIG. 10. Ensemble average of the spatial modes $(\hat{u}, \hat{v}, \hat{w})$ normalized bu $\hat{u}_{\max }$ obtained from LES (solid lines) and theoretical predictions using LSA (dashed lines). Dispersion of the local modes from the LES simulation is highlighted by the shaded grey regions. (a-c) Spatial modes associated with $\lambda_{1}$ in the linear region $\left(\xi / \delta_{0}=7\right)$. (d-f) Spatial modes associated with $\lambda_{2}$ in the turbulent region $\left(\xi / \delta_{0}=40\right)$, with insets showing the spatial modes associated with $\lambda_{2}$ in the linear region, compared to LSA.

LSA modes (insets of figure 11, bottom panels), meaning that $\lambda_{2}$ is present and grows from the beginning of the computational domain. It is found that the local disturbance velocity modes collapse, and a unique spatial mode emerges. In the turbulent region, the spatial modes have a similar shape as those in the linear region but with a relaxation of the profile in the normal direction; more importantly $\hat{u}$ is strictly positive, $\hat{v}$ is strictly negative and $\hat{w}$ is positive near the wall and negative in the outer layer, as it is the case in the initial linear growth region. In terms of amplitude, the observed spatial modes associated with $\lambda_{2}$ have a much larger amplitude in the turbulent region than in the linear region, as expected. The existence of the spatial modes in the turbulent region is thus consistent with the observation of streamwise Görtler-like vortices in the turbulent region reported in figure 5(b). This shows that spatial modes for the disturbance velocity 


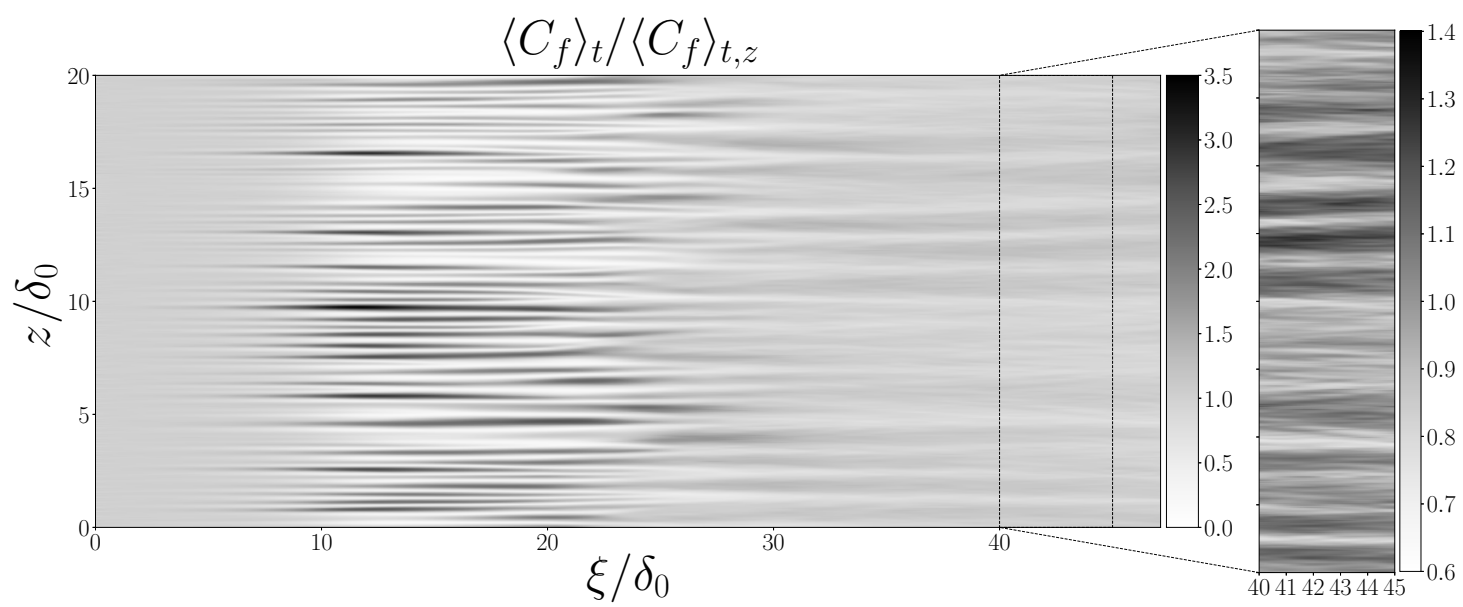

FIG. 11. Curved wall colored by the mean skin friction coefficient $\left\langle C_{f}\right\rangle_{t}$ normalized by the local spanwise averaged skin friction coefficient $\left\langle C_{f}\right\rangle_{t, z}$. The zoomed area in the inset $\left(40<\xi / \delta_{0}<45\right)$ highlights the persistence of the heterogeneity in the fully turbulent region.

converge and can be predicted using laminar LSA even in the fully turbulent region using the turbulent Görtler number instead of the classical $G_{\theta}$.

\section{Skin friction coefficient}

The presence of dominant wavelengths is expected to have an effect on several properties of the flow, especially on the mean wall shear stress $\left\langle\tau_{w}\right\rangle_{t}=\left.\mu \partial_{\psi}\langle u\rangle_{t}\right|_{\psi=0}$ or in its non-dimensional form, the mean skin friction coefficient $\left\langle C_{f}\right\rangle_{t}=\left\langle\tau_{w}\right\rangle_{t} /\left(0.5 \rho U_{\infty}^{2}\right)$.

In figure 11, we show the distribution of $\left\langle C_{f}\right\rangle_{t}$ over the full computational domain normalized with the spanwise-averaged skin friction coefficient $\left\langle C_{f}\right\rangle_{t, z}$ in order to assess the heterogeneity of the friction in the spanwise direction. The development of the Görtler instability clearly leads to a steady spanwise heterogeneity of $\left\langle C_{f}\right\rangle_{t}$ within the linear and non-linear regions $\left(2<\xi / \delta_{0}<22\right)$, as also reported in previous studies ${ }^{12-15,20,22}$. In our study however, this heterogeneity clearly persists also in the turbulent region as highlighted by the inset box in figure $11\left(40<\xi / \delta_{0}<45\right)$. The spanwise fluctuations of $\left\langle C_{f}\right\rangle_{t}$ grow in the linear and non-linear regions $\left(2<\xi / \delta_{0}<22\right)$ up to 3.5 times the local spanwise-average $\left\langle C_{f}\right\rangle_{t, z}$, and up to 1.4 times in the transitional and turbulent regions $\left(\xi / \delta_{0}>22\right)$, i.e. up to $40 \%$ of $\left\langle C_{f}\right\rangle_{t, z}$.

The phase-based statistical treatment given in equation (11) is also applied to the skin friction coefficient $\left\langle C_{f}\right\rangle_{t}$. Figure 12(a) shows the evolution of the mean skin friction coefficient $\left\langle C_{f}\right\rangle_{t, z}$ 


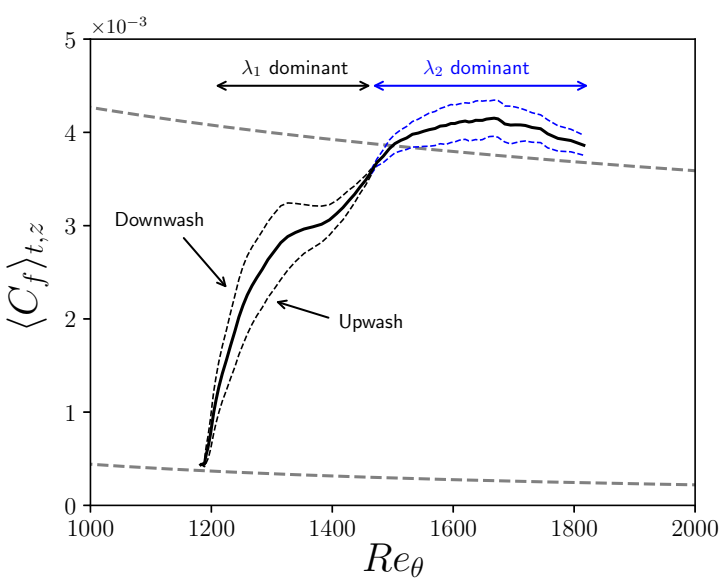

(a)

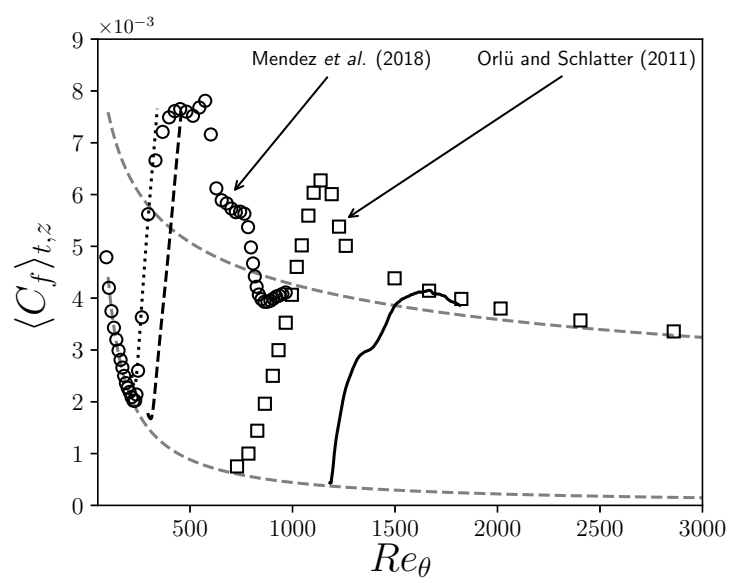

(b)

FIG. 12. (a) Evolution of the spanwise-averaged mean skin friction coefficient $\left\langle C_{f}\right\rangle_{t, z}$ (black solid line) and the local mean skin friction coefficient $\left\langle C_{f}\right\rangle_{t}$ at upwash (lower line) and downwash (upper line) locations with respect to $\lambda_{1}$ (black dashed lines) and $\lambda_{2}$ (blue dashed lines), as a function of $\operatorname{Re}_{\theta}$. Grey dashed lines represent theoretical laminar $\left(C_{f}=0.441 R e_{\theta}^{-1}\right)$ and turbulent $\left(C_{f}=0.024 R e_{\theta}^{-1 / 4}\right)$ laws, respectively, from Smits, Matheson, and Joubert ${ }^{33}$. (b) Comparison with existing data (circles Méndez et al. ${ }^{20} \operatorname{Re}_{\theta_{0}}=75$; squares Örlü and Schlatter ${ }^{23} \operatorname{Re}_{\theta_{0}}=750$ ) and LES simulations at lower $\operatorname{Re}_{\theta_{0}}$ and $G_{0}$ (dashed black line for $\left(\operatorname{Re}_{\theta_{0}}, G_{\theta_{0}}\right)=(210,13.4)$ and dotted black line for $\left.\left(\operatorname{Re}_{\theta_{0}}, G_{\theta_{0}}\right)=(290,18.4)\right)$

(thick solid line) as a function of the Reynolds number $R e_{\theta}$. Laminar and turbulent laws from Smits, Matheson, and Joubert ${ }^{33}$ (dashed grey lines) are also reported for comparison. The evolution of $\left\langle C_{f}\right\rangle_{t}$ at upwash and downwash locations is computed using the reduced streamwise velocity field $\tilde{u}(\xi, \psi, \tilde{z})$. Upwash and downwash locations are defined with respect to the two dominant wavelengths $\lambda_{1}$ and $\lambda_{2}$ represented in figure 12 as dotted lines. The skin friction coefficient $\left\langle C_{f}\right\rangle_{t, z}$ detaches quickly from the laminar solution at the inflow (lower black dashed line) due to the high initial imposed Reynolds number $\operatorname{Re}_{\theta, 0}=1,175$. In the linear region $\left(1175<\operatorname{Re}_{\theta}<1220\right)$, with the dominant wavelength $\lambda_{1}$, upwash and downwash effects start to appear. In the following nonlinear region, $\left\langle C_{f}\right\rangle_{t, z}$ upwash and downwash effects both increase noticeably. When saturation occurs, upwash and downwash effects slowly diminish, and $\left\langle C_{f}\right\rangle_{t}$ is found to be homogeneous with respect to $\lambda_{1}$.

The rapid onset of the second dominant wavelength $\lambda_{2}$ after ceasing of $\lambda_{1}$, induces a nonhomogeneous distribution of $\left\langle C_{f}\right\rangle_{t}$ in both the transition and turbulent regions. Upwash and downwash effects with respect to $\lambda_{2}$ start to appear right after the transition to turbulence, at 
$R e_{\theta}=1,500$, and do not vanish within the limits of the present computational domain. The values of $\left\langle C_{f}\right\rangle_{t, z}$ approach and slightly exceed the predictions for the turbulent boundary layer over a flat plate $^{33}$ in the final part of the computational domain (figure 12).

This is very different from what has been reported before ${ }^{7,13,14,20}$ at lower Reynolds $R e_{\theta}$ and Görtler $G_{\theta}$ numbers. In those studies, the spanwise-averaged skin friction coefficient $\left\langle C_{f}\right\rangle_{t, z}$ in the non-linear region clearly overshoots the theoretical predictions for a turbulent boundary layer over a flat plate and the skin friction coefficient is found to be larger already in the transition region corresponding to the domain of existence of the first unstable mode $\lambda_{1}$. Here, $\left\langle C_{f}\right\rangle_{t, z}$ slightly exceeds the turbulent prediction only for the second dominant wavelength of the Görtler instability in the turbulent domain, as shown in figure 12a. We compare our results with those of Méndez et al. ${ }^{20}$, Örlü and Schlatter ${ }^{23}$ that are represented as squares and circles in figure 12(b), respectively. We also report results from additional two simulations we performed in which we fixed the initial Reynolds number $R e_{\theta}=210$ (light grey line) and $R e_{\theta}=290$ (dark grey line), keeping all other conditions identical. Our results at lower initial $\operatorname{Re}_{\theta}$ follow those of Méndez et $a l .{ }^{20}$ and we also report an overshoot of the skin friction coefficient for the first dominant wavelength (cf. also Ducoin, Shadloo, and Roy ${ }^{7}$ ).

The high value of initial $R e_{\theta}$ induce non-linear effects that act primarily on smaller wavelengths, i.e. on the first appearing Görtler wavelength $\lambda_{1}$. This induces a fast damping of the wavelength $\lambda_{1}$ resulting in a different behaviour for the skin friction coefficient $C_{f}$, where an overshoot of the turbulent plate prediction is not observed for the first appearing unstable Görtler wavelength. The results of Örlü and Schlatter ${ }^{23}$ for large initial $\operatorname{Re}_{\theta_{0}}$ report an overshoot of $C_{f}$ during transition to turbulence. Here, the rapid onset of the second wavelength $\lambda_{2}$ allows $C_{f}$ continuing increasing until it reaches and slightly exceeds the flat plate turbulent prediction, while approaching the flat plate turbulent law at the end of the computational domain.

Besides the different effects induced by initial conditions (such as tripping methods), the presence of an overshoot for the skin friction coefficient $C_{f}$ appears to depend on the curvature $\sqrt{\theta / R}$ for a fixed value of the Görtler number $G_{\theta}$, as reported in Méndez et al. ${ }^{20}$ : they observed a diminution of the overshoot of $C_{f}$ at a given Görtler number when the radius of curvature $R$ was increased. 


\section{SUMMARY AND CONCLUSIONS}

A Large Eddy Simulation of a boundary layer flow over a concave wall has been performed using a Blasius inflow profile and without turbulence and wavelength forcing. The inlet Reynolds and Görtler numbers are $\operatorname{Re}_{\theta_{0}}=1,175$ and $G_{\theta_{0}}=75$, respectively. Transition to turbulence is induced by the natural development of the Görtler instability, and a developed turbulent region is reached at the end of the computational domain, with maximum $\operatorname{Re}_{\theta}=1,800$ and $G_{\theta}=140$.

The developed flow over a concave wall exhibits steady large scale vortical structures that induce a spanwise heterogeneity of the mean flow properties even in the developed turbulent region, with a first clear wavelength in the initial development region $\lambda_{1}=0.385 \delta_{0}$ and a second clear wavelength $\lambda_{2}=1.55 \delta_{0}$ in the turbulent region.

The predictions of both the most amplified wavelength along with the associated spatial modes obtained by extending the LSA of Floryan and Saric $^{3}$ to a wider parameter domain of $G_{\theta}$ and the non-dimensional wavelength $\Lambda$ well compare to the computed wavelength and the spanwise averaged spatial modes in the linear region for $\lambda_{1}$. The dominant wavelength $\lambda_{2}$ in the turbulent domain is well predicted with the LSA if a turbulent Görtler number is considered, in which the kinematic viscosity $v$ is replaced by the turbulent viscosity $v_{t}$, as proposed by Tani ${ }^{11}$. Also, the spatial modes associated with $\lambda_{2}$ converge with a low scatter in the turbulent region.

The skin friction coefficient $\left\langle C_{f}\right\rangle_{t}$ increases locally up to a factor 3.5 in the non-linear region of dominance of $\lambda_{1}$ and up to a factor of 1.4 in the developed turbulent region for the downwash location of the $\lambda_{2}$ Görtler instability, very different from the homogeneous distribution of $\left\langle C_{f}\right\rangle_{t}$ reported previously in the literature, in which no Görtler vorticies develop in the turbulent region because the estimated turbulent Görtler number is close or even below the neutral stability curve. Thus, the flow in the turbulent region becomes Görtler stable. The high value of initial $\operatorname{Re}_{\theta}$ induce non-linear effects that act primarily on the first appearing Görtler wavelength $\lambda_{1}$ damping its growth, so that the skin friction coefficient $C_{f}$ slightly exceeds the flat plate turbulent prediction for the second dominant wavelength $\lambda_{2}$ at the end of the computational domain, and no overshoot of $C_{f}$ is reported in the region of dominance of $\lambda_{1}$. Additional simulations we performed with a smaller initial $\operatorname{Re}_{\theta, 0}$ are in accord with previous studies with an overshoot of $C_{f}$ for the first dominant Görtler wavelength with respect to the turbulent plate predictions. 


\section{ACKNOWLEDGMENTS}

This work has been supported by a grant from Labex OSUG (Investissements d'avenir ANR10 LABX56). V. Moureau and G. Lartigue (CORIA), and the SUCCESS scientific group are acknowledged for providing the YALES2 code. This work was granted access to the HPC resources of IDRIS under the allocation 2019-A0060107567 made by GENCI.

Special thanks go to E.J. Hopfinger for fruitful discussions.

\section{DATA AVAILABILITY STATEMENT}

The data that support the findings of this study are available from the corresponding author upon reasonable request.

\section{REFERENCES}

${ }^{1}$ H. Görtler, "Über eine dreidimensionale Instabilität laminarer Grenzschichten an konkaven Wänden,” Nachr. Ges. Wiss. Göttingen, NF 2, 1-26 (1940).

${ }^{2}$ A. Smith, "On the growth of taylor-görtler vortices along highly concave walls," Quart. App. Math. 13, 233-262 (1955).

${ }^{3}$ J. Floryan and W. Saric, "Stability of görtler vortices in boundary layers," AIAA journal 20, 316-324 (1982).

${ }^{4} \mathrm{P}$. Hall, “The linear development of görtler vortices in growing boundary layers," J. Fluid Mech. 130, 41-58 (1983).

${ }^{5}$ W. Saric, “Görtler vortices,” Ann. Rev. Fluid Mech. 26, 379-409 (1994).

${ }^{6} \mathrm{~S}$. Navarro-Martinez and O. Tutty, "Numerical simulation of görtler vortices in hypersonic compression ramps," Comp. \& Fluids 34, 225-247 (2005).

${ }^{7}$ A. Ducoin, M. Shadloo, and S. Roy, "Direct numerical simulation of flow instabilities over savonius style wind turbine blades," Ren. Ener. 105, 374-385 (2017).

${ }^{8}$ E. Hopfinger, A. Kurniawan, W. Graf, and U. Lemmin, "Sediment erosion by görtler vortices: the scour-hole problem," J. Fluid Mech. 520, 327-342 (2004).

${ }^{9}$ I. Albayrak, E. Hopfinger, and U. Lemmin, "Near-field flow structure of a confined wall jet on flat and concave rough walls," J. Fluid Mech. 606, 27-49 (2008). 
Linear to turbulent Görtler instability transition

${ }^{10} \mathrm{C}$. Brun, "Large-eddy simulation of a katabatic jet along a convexly curved slope: 2 . evidence of görtler vortices,” J. Geophys. Res. Atm. 122, 5190-5210 (2017).

${ }^{11}$ I. Tani, "Production of longitudinal vortices in the boundary layer along a concave wall," J. Geophys. Res. 67, 3075-80 (1962).

12J. Swearingen and R. Blackwelder, "The growth and breakdown of streamwise vortices in the presence of a wall," J. Fluid Mech. 182, 255-290 (1987).

${ }^{13}$ T. Tandiono, S. Winoto, and D. Shah, "Spanwise velocity component in nonlinear region of görtler vortices," Phys. of Fluids 25, 104104 (2013).

${ }^{14}$ L.-U. Schrader, L. Brandt, and T. Zaki, "Receptivity, instability and breakdown of görtler flow," J. Fluid Mech. 682, 362-396 (2011).

${ }^{15}$ V. Malatesta, L. Souza, and J. Lui, "Influence of goertler vortices spanwise wavelength on heat transfer rates," Comp. Therm. Sci. 5, 389-400 (2013).

${ }^{16}$ L. Souza, "On the odd and even secondary instabilities of görtler vortices," Theoretical and Computational Fluid Dynamics 31, 405-425 (2017).

${ }^{17}$ M. B. J. M. P. A. N. H. M. S. A. J. Marusic, I. and K. R. Sreenivasan, "Wall-bounded turbulent flows at high reynolds numbers: Recent advances and key issues.” Phys. Fluids , 065103 (2010).

${ }^{18}$ P. Schlatter, Q. Li, G. Brethouwer, A. Johansson, and D. Henningson, "Structure of a turbulent boundary layer studied by dns," in Direct and Large-Eddy Simulation VIII (Springer, 2011) pp. 9-14.

${ }^{19}$ P. Schlatter and R. Örlü, "Inflow length and tripping effects in turbulentboundary layers," J. Phys.: Conf. Ser. 318, 022018 (2011).

${ }^{20}$ M. Méndez, M. Shadloo, A. Hadjadj, and A. Ducoin, "Boundary layer transition over a concave surface caused by centrifugal instabilities," Computers \& Fluids 171, 135-153 (2018).

${ }^{21}$ D. Park and P. Huerre, "Primary and secondary instabilities of the asymptotic suction boundary layer on a curved plate," J. Fluid Mech. 283, 249-272 (1995).

${ }^{22}$ M. Schultz and R. Volino, "Effects of concave curvature on boundary layer transition under high freestream turbulence conditions," J. Fluids Eng. 125, 18-27 (2003).

${ }^{23}$ R. Örlü and P. Schlatter, "Inflow length and tripping effects in turbulent boundary layers," in Journal of Physics: Conference Series, Vol. 318 (IOP Publishing, 2011) p. 022018.

${ }^{24}$ V. Moureau, P. Domingo, and L. Vervisch, "Design of a massively parallel cfd code for complex geometries," Comptes Rendus Mécanique 339, 141 - 148 (2011), high Perf. Comp. 
Linear to turbulent Görtler instability transition

${ }^{25}$ A. Chorin, "Numerical solution of the navier-stokes equations," Math. of Comp. 22, 745-762 (1968).

${ }^{26}$ M. Malandain, N. Maheu, and V. Moureau, "Optimization of the deflated conjugate gradient algorithm for the solving of elliptic equations on massively parallel machines," J. Comp. Phys. 238, 32 - 47 (2013).

${ }^{27}$ M. Germano, U. Piomelli, P. Moin, and W. Cabot, “A dynamic subgrid-scale eddy viscosity model," Phys. of Fluids 3, 1760-1765 (1991).

${ }^{28}$ I. Orlanski, “A simple boundary condition for unbounded hyperbolic flows,” Journal of computational physics 21, 251-269 (1976).

${ }^{29}$ S. B. Pope, "Turbulent flows," (2001).

${ }^{30}$ P. Benard, G. Balarac, V. Moureau, C. Dobrzynski, G. Lartigue, and Y. D’ Angelo, “Mesh adaptation for large-eddy simulations in complex geometries," International Journal for Numerical Methods in Fluids 81, 719-740 (2016).

${ }^{31} \mathrm{P}$. Petitjeans, Étude expérimentale des instabilités de couches limites sur des parois concaves : Instabilité de Görtler, Phd thesis (1992).

${ }^{32}$ F. H. Clauser, "The turbulent boundary layer," Adv. Appl. Mech. 4, 1-51 (1956).

${ }^{33}$ A. Smits, N. Matheson, and P. Joubert, "Low-reynolds-number turbulent boundary layers in zero and favorable pressure gradients," Journal of ship research 27, 147-157 (1983). 